\title{
XXXVIII.
}

\section{THE ACTUAL CAUTERY IN THE TREATMENT OF LOCALIZED TUBERCULOUS LESIONS.}

\author{
By GEORge B. WoOd, M. D.,
}

\section{Philadelphia.}

For some time I have felt that the actual cautery, and preferably the platinum wire heated by electricity, is by far the most effective method of combating tuberculosis of the upper respiratory tract. The striking results which have followed its use in cases of pharyngeal tuberculosis have confirmed my belief in its efficiency. If a pharyngeal lesion is not too extensive and can be reached by the heat, I am of the opinion that if treated with the cautery it will in all cases heal promptly, at least such has been my experience.

The good results following the use of the cautery are equally great in the larynx, provided the lesion is so placed as to make it accessible to the action of the heat. The beneficial action of the cautery seems to reach distinctly beyond the area of destruction, and the resulting scar does not appear to be easily reinvaded.

Last fall I removed with the cautery snare for diagnostic purposes, a suspicious looking tonsil from an arrested case of pulmonary tuberculosis. The portion of the tonsil removed -showed extensive typical tonsillar tuberculosis, consisting of minute young tubercles scattered throughout its extent. Five days later I removed the rest of the tonsil. The result of this last piece of tonsil was most interesting and led me to make a small series of experiments on guinea pigs. The scar in the portion of tonsil removed was surprisingly large and apparently already made up of well developed fibrous tissue, and only after the examination of a considerable number of sections did I finally discover a single small miliary tubercle, and this seemed to be already undergoing fibroid change. 
EXPERIMENTS ON GUINEA PIGS.

The object of these experiments was to determine the histologic appearance of a cautery wound made through a tuberculous lesion. The series is somewhat short, but demonstrates certain peculiarities in the healing of the wound which are important in relation to the cure of a localized tuberculous lesion. It was desired to produce a superficial localized tuberculous lesion. For this purpose an attenuated culture of human tubercle bacilli which had been grown through five generations on artificial media was used. The culture from which the bacilli were taken was three weeks old.

On February 15th one c.c. of an emulsion of the above culture was injected just beneath and into the skin of the abdomen of three guinea pigs. Each pig was inoculated on each side of the abdomen so as to make two distinct and separate lesions.

On March 7th pig No. 1 showed marked induration of the inoculated areas, and on the left side there was some sloughing in the center of the lesion. About three-fourths of the lesion was cut out on the left side, and about half the lesion on the right side was destroyed with the electric cautery. The piece of skin removed from the left side was hardened in Zenker's solution imbedded in paraffin and sections stained with hematoxylin and eosin. These sections showed a large central lesion of epitheloid cells with slight necrosis in the center ; surrounding this were a few isolated discrete tubercles composed of epitheloid cells. There was very little tendency to the formation of giant cells and there were numerous polymorphonuclear cells scattered throughout the central lesions and massing on the surface.

On March 13th the animal was killed, the lesions removed and hardened in Zenker's solution. Sections were stained with hematoxylin and eosin and also with Mallory's connective tissue stain. Macroscopically the lesion which had been cut showed advance and the skin was apparently adherent to the abdominal muscles, but there were no tubercles on the neighboring peritoneum. The burnt lesion macroscopically appeared to be healing and was not adherent to the underlying muscle tissue.

Under the microscope the cut lesion showed the ordinary 
histologic appearance of tuberculosis without the formation of giant cells. There was considerable necrosis, some extension into the surrounding muscle tissue and some new fibrous tissue. There were a few new-formed blood vessels; but no marked inflammatory reaction.

The burned lesions showed considerable tuberculous tissue, but around the margin where the slough following the cauterization had come away there was a marked inflammatory reaction. There were numerous new blood vessels, most of them were filled with blood, and the formation of new fibrous tissue was very marked. Outside of this zone of reaction the tuberculous tissue appeared apparently unaltered, and there were some tubercles in the surrounding muscle.

Pig No. 2. March 28th cauterized both abdominal lesions.

March 31st. Pig killed. The skin lesions were removed and hardened in Zenker's solution. Sections were stained in hematoxylin and eosin. The inguinal lymph glands were enlarged and caseous. On both abdominal lesions the eschar was still adherent and there seemed to be a slight shrinking in size of the tuberculous area since they had been burned.

Microscopically the lesion showed a considerable area of tuberculosis, unaltered except in a zone surrounding the base of the eschar. Here the inflammatory reaction was fairly well marked and the new blood vessels, while not so numerous as in the burned lesion of pig No. 1, formed a distinct zone, many of them being filled with blood. There was some tuberculous infiltration of the surrounding muscle tissue.

Pig No. 3. March 26th cauterized both abdominal lesions and punctured an enlarged right inguinal lymph gland with cautery point. The node was completely caseous, a large quantity of the material escaping when the platinum point went through the capsule.

April 17th. Pig killed. The lesions in the abdominal wall and the left inguinal lymph gland were removed and placed in Zenker's solution. Sections were stained with hematoxylin and eosin and Mallory's connective tissue stain. Macroscopically the lesion on the left side of the abdomen showed a healthy, shallow, raw surface without induration, and probably was caused by breaking a partially healed wound by rough handling. The wound in the right side of the abdomen was so nearly healed that it was difficult to find until the hair had been re- 
moved. There was a slight induration along the scar, and at one end thete was a small crust, which showed the wound had not completely healed. There was a beginning general miliary tuberculosis.

Histologically the left wound appeared as a shallow ulcer with a small amount of necrosis on the surface and numerous polymorphonuclear leucocytes. There was some tuberculous tissue in the immediate neighborhood, as evidenced by the presence of epitheloid cells. The right wound showed a healed surface with a rather large deposit of fibrous tissue, but underneath this a small area of epitheloid cells. The inguinal lymph gland showed a small, healed punctate scar, but it was so completely caseous that the histologic study did not show anything of interest.

\section{SUMMARY.}

In three days after the cauterization of a low grade tuberculous lesion there developed a distinct zone of inflammatory reaction around the area of destruction. This reaction was manifest by the presence of newly formed blood vessels, congestion and fibroblasts. Beyond this zone of reaction the tuberculous process seemed unaltered. In six days the zone of reaction was much more marked, the blood vessels being more numerous and larger, and there was a distinct deposit of fibrous tissue between the epitheloid cells of the tuberculous mass. The tuberculous process beyond the zone of reaction was apparently unaltered except that it seemed for some reason less in extent. In twenty days the lesions were to the naked eye almost healed. A fibrous cicatrix had formed in the zone of the inflammatory reaction, and though there was some tuberculous tissue still present beneath the scar, the amount was very small and becoming gradually cicatrized.

These few experiments seem to have substantiated certain clinical observations as regard to the use of the cautery in treating localized tuberculosis. There is no doubt that a retarding influence is exerted beyond the area actually destroyed by the heat. We know that a tuberculous nodule heals by cicatrization, and therefore anything that aids in the formation of fibrous tissue aids in the cure of the disease. Further, by the induction of acute inflammation with the subsequent formation of new blood vessels, nutrition is brought 
to a part which would otherwise break down into a necrotic mass because of a lack of sufficient food to nourish the tissues. Again, the eschar produced by the burning prevents reinoculation until the tissue has become sufficiently resistant to protect itself. Also the cauterization seals the lymphatics and blood vessels, thereby preventing a spread of the disease either locally through the lymph system or generally through the blood vessels. 